ARTICLE

DOI: $10.1038 / \mathrm{s} 41467-018-03289-7$

\title{
TET-mediated epimutagenesis of the Arabidopsis thaliana methylome
}

\author{
Lexiang Ji ${ }^{1}$, William T. Jordan², Xiuling Shi ${ }^{2}$, Lulu Hu${ }^{3,4,5}$, Chuan He (i) ${ }^{3,4,5}$ \& Robert J. Schmitz ${ }^{2}$
}

DNA methylation in the promoters of plant genes sometimes leads to transcriptional repression, and the loss of DNA methylation in methyltransferase mutants results in altered gene expression and severe developmental defects. However, many cases of naturally occurring DNA methylation variations have been reported, whereby altered expression of differentially methylated genes is responsible for agronomically important traits. The ability to manipulate plant methylomes to generate epigenetically distinct individuals could be invaluable for breeding and research purposes. Here, we describe "epimutagenesis," a method to rapidly generate DNA methylation variation through random demethylation of the Arabidopsis thaliana genome. This method involves the expression of a human ten-eleven translocation (TET) enzyme, and results in widespread hypomethylation that can be inherited to subsequent generations, mimicking mutants in the maintenance of DNA methyltransferase met1. Application of epimutagenesis to agriculturally significant plants may result in differential expression of alleles normally silenced by DNA methylation, uncovering previously hidden phenotypic variations.

\footnotetext{
${ }^{1}$ Institute of Bioinformatics, University of Georgia, Athens, GA 30602, USA. ${ }^{2}$ Department of Genetics, University of Georgia, Athens, GA 30602 , USA.

${ }^{3}$ Department of Chemistry, University of Chicago, Chicago, IL 60637, USA. ${ }^{4}$ Department of Biochemistry and Molecular Biology, University of Chicago, Chicago, IL 60637, USA. ${ }^{5}$ Howard Hughes Medical Institute, University of Chicago, Chicago, IL 60637, USA. These authors contributed equally: Lexiang Ji and William T. Jordan. Correspondence and requests for materials should be addressed to R.J.S. (email: schmitz@uga.edu)
} 
O ur ability to develop novel beneficial crop traits has significantly improved over the last 100 years, although the ability to maintain this trajectory is limited by allelic diversity. While genetic variation has been heavily exploited for crop improvement, utility of epigenetic variation has yet to be efficiently implemented. Epigenetic variation arises not from a change in the DNA sequence, but by changes in modifications to DNA such as cytosine methylation. This variation can result in the emergence of novel and stably inherited phenotypes, as well as unique patterns of gene expression.

In plant genomes, cytosine methylation occurs at three major sequence contexts: $\mathrm{CG}, \mathrm{CHG}$, and $\mathrm{CHH}$ (where $\mathrm{H}=\mathrm{A}, \mathrm{C}$, or $\mathrm{T})^{1}$. Methylation at these different contexts is coordinated by distinct maintenance mechanisms during DNA replication. The methylation of DNA in all three contexts is essential for transcriptional silencing of transposons, repeat sequences, and certain genes. Genes regulated by this mechanism are stably repressed throughout the soma and represent an untapped source of hidden genetic variation if transcriptionally re-activated, as revealed from pioneering studies in the model plant $A$. thaliana ${ }^{2-4}$. However, the impact of this variation is not observed in wild-type plants, as genes silenced by DNA methylation are not expressed. This novel source of genetic variation was uncovered by creating epigenetic recombinant inbred lines (epiRILs) from crosses between a wildtype individual and a mutant defective in maintenance of DNA methylation $^{2-4}$. EpiRILs, while genetically wild type, contain mosaic DNA methylomes dependent on chromosomal inheritance patterns, as DNA methylation is meiotically inherited in $A$. thaliana $^{2,}{ }^{5-8}$. Phenotypic characterization of epiRILs has revealed extensive morphological variation with respect to traits such as flowering time, root length, and resistance to bacterial infection $^{2-4}$. The morphological variation generated by the creation of epiRILs has revealed extensive hidden genetic variation in plant genomes that can be observed due to expression of newly unmethylated regions. However, the creation of epiRILs requires one founding parent to be a null mutant in the maintenance DNA methylation pathway. Unfortunately, unlike in $A$. thaliana, the loss of DNA methylation maintenance activity often results in lethality in crops 9,10 . Therefore, novel methodologies are required to realize the potential of these hidden epialleles in crop genomes.

Epimutagenesis is an alternative method to generate epiRILs. Instead of relying on the genome-wide demethylation of one of the two founding parents, epimutagenesis introduces random methylation variation via the introduction of a transgene. Here, we describe a novel epimutagenesis approach in A. thaliana using a human ten-eleven translocation methylcytosine dioxygenase 1 (TET1) ${ }^{11-14}$, which catalyzes the conversion of 5-methylcytosine $(5 \mathrm{mC})$ to 5-hydroxymethylcytosine $(5 \mathrm{hmC})$. Although TET enzymes and their primary product $5 \mathrm{hmC}$ are not found in plant genomes $^{15}$, ectopic expression of a human TET enzyme resulted in widespread DNA demethylation and induced phenotypic variation in $A$. thaliana.

\section{Results}

Overexpressing TET1 in Arabidopsis hypomethylates the genome. Transgenic A. thaliana plants were generated expressing the catalytic domain (residues 1418-2136) of the human TET1 protein (hTET1cd) under the control of the CaMV35S promoter. To assess the impact of hTET1cd expression on the A. thaliana methylome, whole-genome bisulfite sequencing (WGBS) was performed on two independently derived transgenic plants (35S: TET1-1 and 35S:TET1-2; Supplementary Table 1). WGBS revealed a global reduction of CG methylation from $18.2 \%$ in two wild-type individuals to $8.9 \%$ in $35 \mathrm{~S}$ :TET1-1 and $6.9 \%$ in $35 \mathrm{~S}$ :
TET1-2 (compared to $0.5 \%$ in met $1-3$ ). The effects of hTET1cd expression on $\mathrm{CHG}$ and $\mathrm{CHH}$ methylation were not as severe compared to CG methylation (Fig. 1a). Importantly, different degrees of CG hypomethylation were observed in different independent transgenic plants. This result has important implications for epimutagenesis in economically and agriculturally significant plant species, as it appears feasible to control the degree of DNA hypomethylation by screening for plants with desired levels of demethylation. Taken together, these results show that the expression of hTET1cd results in intermediate CG methylation levels when compared to wild-type and met1 individuals.

The primary product of TET1 oxidation is $5 \mathrm{hmC}$, which is indistinguishable from $5 \mathrm{mC}$ by WGBS. We therefore performed Tet-assisted bisulfite sequencing (TAB-seq) to profile $5 \mathrm{hmC}$ levels in 35S:TET1 plants ${ }^{16}$. No detectable levels of $5 \mathrm{hmC}$ were found in the transgenic lines assayed (Supplementary Fig. 1a, b). Thus, the widespread loss of CG DNA methylation observed may result from a failure to maintain methylation at CG sites that possess $5 \mathrm{hmC}$, or through active removal of $5 \mathrm{hmC}$ or further oxidized products via the base excision repair pathway.

To better understand the effects of hTET1cd expression, we determined changes in the $A$. thaliana methylome at the chromosomal and local levels. Plotting methylation levels across all five chromosomes revealed a strong depletion of CG methylation at the pericentromeric region (Fig. 1b). CG hypomethylation occurred at both gene body methylated (gbM) and select RNA-directed DNA methylated (RdDM) loci. (Fig. 1c, d). To further quantify the observed hypomethylation, metaplots were created for genes and transposons, respectively (Fig. 1e, $\mathrm{f}$ and Supplementary Fig. $1 \mathrm{c}-\mathrm{f})$. A strong reduction of $\mathrm{mCG}$ and a mild reduction of $\mathrm{mCHG} / \mathrm{mCHH}$ were observed at both genes and transposons. On average, $97.9 \%$ of gbM genes and $56.7 \%$ of methylated transposons (where these regions have at least $50 \%$ mCG in wild type) lost at least half of their CG methylation in epimutagenized lines (Supplementary Data 1 and 2). Collectively, these results indicate that hypomethylation was more severe in genes than transposons, possibly the result of de novo methylation by the $\mathrm{RdDM}$ pathway, which is primarily active at transposons.

TET1-mediated DNA demethylation mimics met1 mutants. An analysis of differentially methylated regions (DMRs) was then carried out to assess the genome-wide impact of hTET1cd expression. A total of 56,283 CG DMRs ranging in size from 6-20,286 base pairs (bp) were identified (Fig. 1g and Supplementary Data 3). Of these, $38.7 \%$ were located in intergenic sequences, $53.7 \%$ overlapped with genes, and $7.6 \%$ were located in promoter regions ( $\leq 1 \mathrm{~kb}$ upstream of a gene). As also seen in met 1 mutants, the predominant effect of hTET1cd expression is CG hypomethylation (12,641 and 20,601 DMRs lost more than 50\% mCG in 35S:TET1-1 and 35S:TET1-2, respectively; no region gained more than 50\% mCG). However, the extent of CG methylation loss caused by hTETcd expression is lower than in met 1: $31.8 \mathrm{Mb}$ of the genome significantly lost CG methylation in met1, whereas $9.9 \mathrm{Mb}$ and $18.0 \mathrm{Mb}$ were lost in 35S:TET1-1 and 35S:TET1-2, respectively.

Previous studies of the met 1 methylome have revealed a loss of $\mathrm{mCHG} / \mathrm{mCHH}$ methylation in a subset of CG-hypomethylated regions ${ }^{17}$. At these loci, DNA methylation is stably lost, in contrast to regions where DNA methylation is re-established by de novo methylation pathways. These loci are ideal targets of epimutagenesis, as the co-existence of all three types of methylation is more frequently correlated with transcriptional repression of genes than CG methylation alone. This, coupled 
a

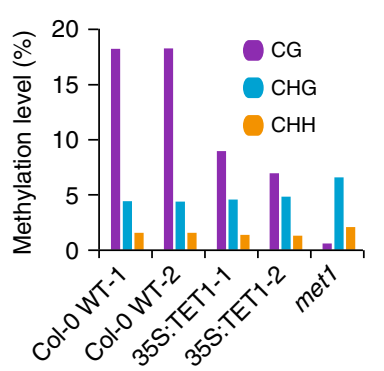

C

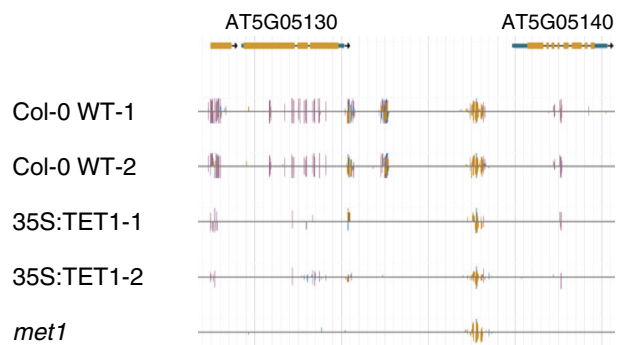

e

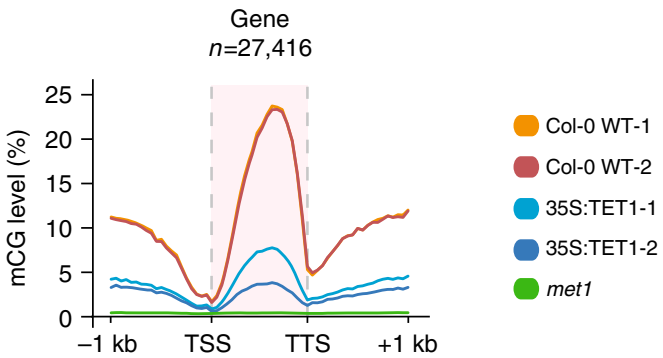

g mCG level (\%) $\begin{array}{llllll}0 & 20 & 40 & 60 & 80 & 100\end{array}$

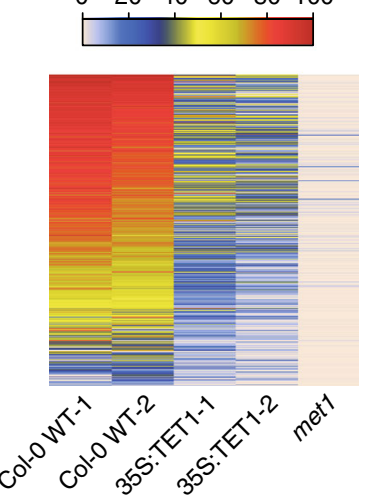

h

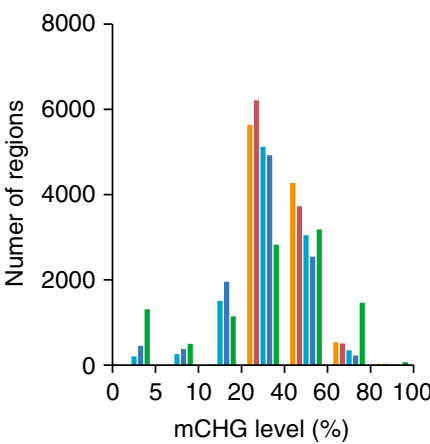

b

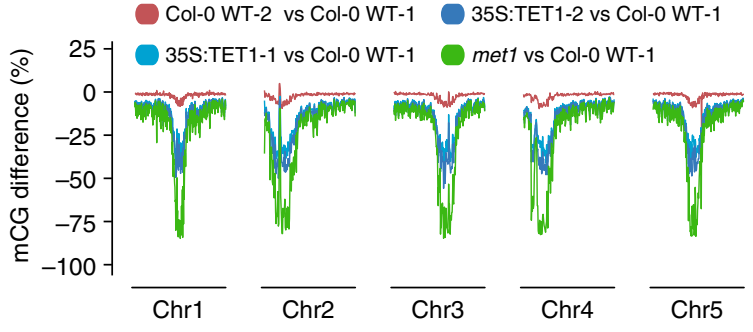

d

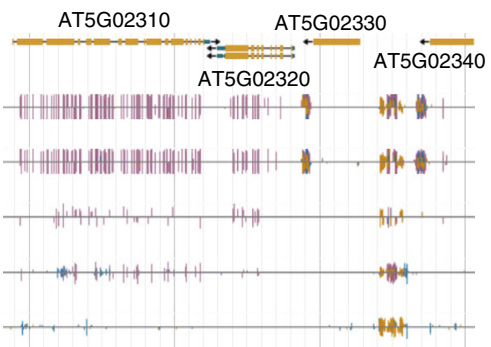

$\mathbf{f}$

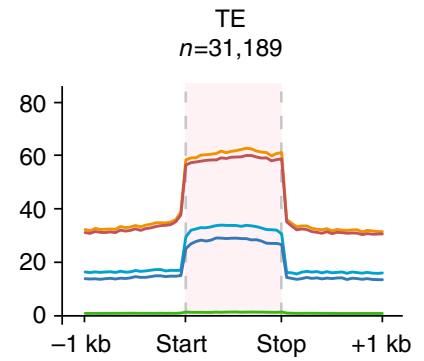

i

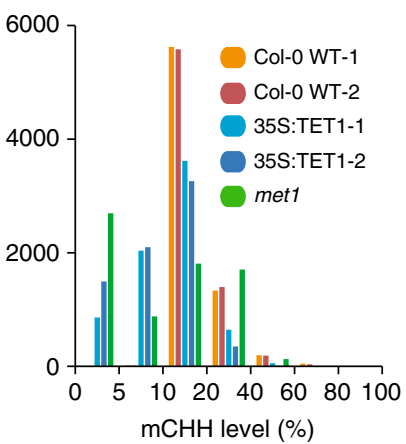

Fig. 1 Overexpression of hTET1cd-induced global CG demethylation in A. thaliana. a Bar graph of global methylation levels in two Col-0 WT replicates, two 35S:TET1 transgenic individuals, and met1. b Metaplot of CG methylation levels (100 kb windows) across five A. thaliana chromosomes. Methylation level differences were defined relative to Col-0 WT-1, and Col-0 WT-2 was used to assess background interference. Genome browser view of methylome profile of two regions $(\mathbf{c}, \mathbf{d})$ of the $A$. thaliana genome (purple vertical lines $=\mathrm{CG}$ methylation, blue vertical lines $=\mathrm{CHG}$ methylation, and gold vertical lines $=\mathrm{CHH}$ methylation). Metagene plots of CG methylation level across e gene bodies and $\mathbf{f}$ transposable elements. $\mathbf{g}$ Heat map of CG methylation level of CG DMRs. Bar plots of $\mathrm{CHG}(\mathbf{h})$ and $\mathrm{CHH}(\mathbf{i})$ methylation levels of CG DMRs that possess non-CG methylation in wild-type individuals

with the long-term stability of hypomethylation, may facilitate inherited transcriptional changes. An analysis of the interdependence of the loss of CG methylation on non-CG methylation levels revealed that $39.7 \mathrm{~Kb}$ and $931.5 \mathrm{~Kb}$ of $\mathrm{CHG}$ methylated sequences lost significant amounts of methylation in two independent epimutagenized lines, compared to $4.0 \mathrm{Mb}$ of sequence in met1 mutants. A similar analysis for the loss of $\mathrm{CHH}$ methylation revealed losses of $23.3 \mathrm{~Kb}$ and $492.5 \mathrm{~Kb}$ in epimutagenized individuals, compared to $1.1 \mathrm{Mb}$ lost in met 1 mutants. Of the 56,283 identified CG DMRs, 10,491 overlapped 
a

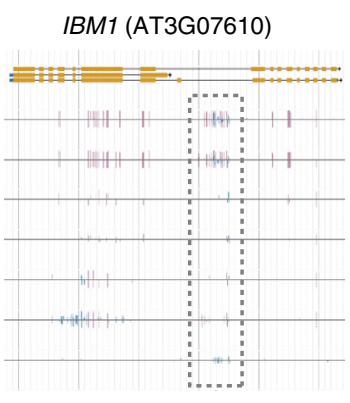

C

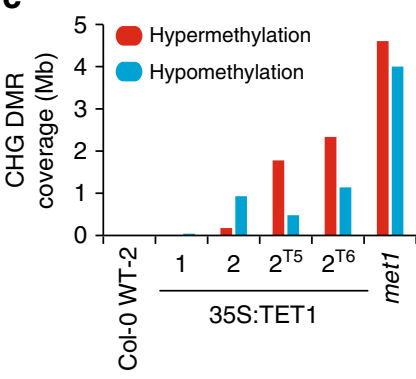

e

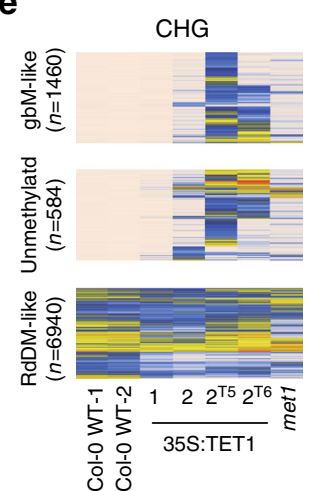

f b

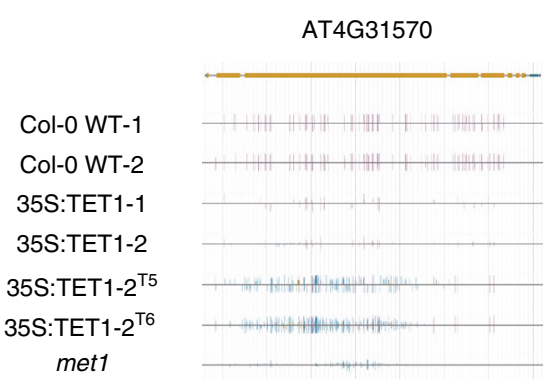

d
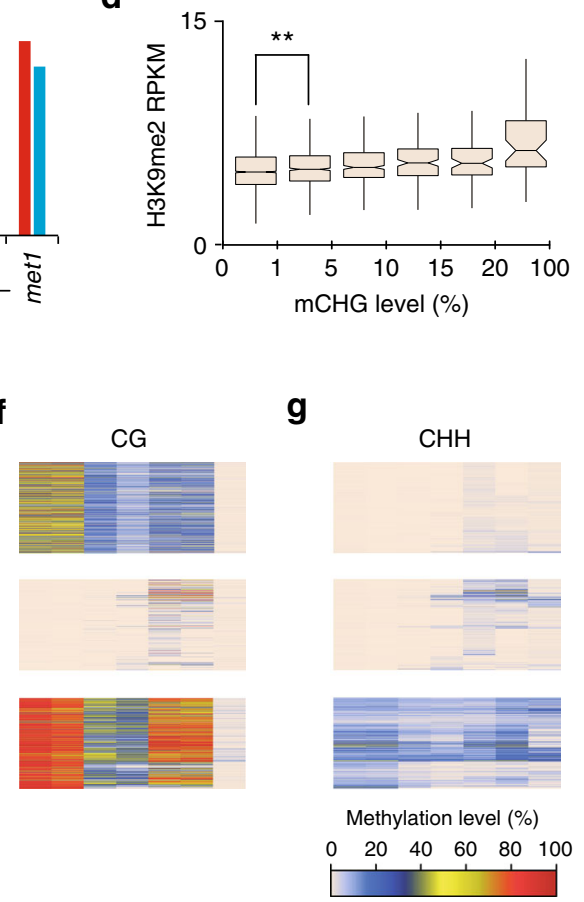

g

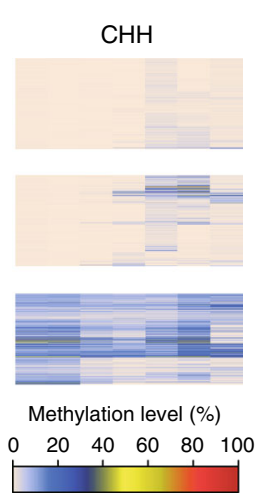

h

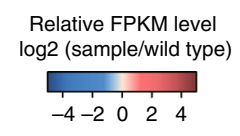

Upregulated

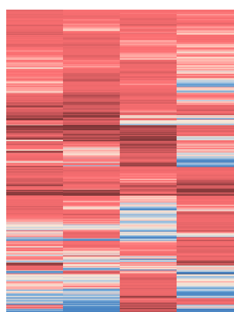

i

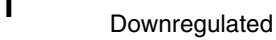

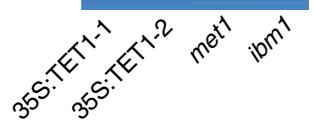

Fig. 2 Global fluctuation of CHG methylation in 35S:TET1 plants. a Genome browser view of IBM1 (AT3G07610) in Col-0 WT, four 35S:TET1 transgenic plants, and met1. A decrease in CG methylation from coding regions was accompanied by an increase in non-CG methylation. Both CG and non-CG methylation were lost from the large intron (purple vertical lines $=\mathrm{CG}$ methylation, blue vertical lines $=\mathrm{CHG}$ methylation, and gold vertical lines $=\mathrm{CHH}$ methylation). b Genome browser view of a representative CHG hypermethylated region. c The amount of the genome affected by differential CHG methylation. These DMRs were defined relative to Col-0 WT-1, as Col-0 WT-2 DMRs were used to assess background interference. $\mathbf{d}$ Boxplot of H3K9me2 distribution in gbM loci (**t-test, $p$ value $<0.01$ ). e Heat map of CHG methylation displaying CHG DMRs. Corresponding CG and CHH methylation levels are shown in $\mathbf{f}$ and $\mathbf{g}$. Heat maps showing $\log _{2}$ transformed FPKM profiles of upregulated genes (h) and downregulated genes (i) in two 35S:TET1 transgenic individuals, met1, and ibm 1 mutants relative to WT

regions that contained at least 20\% CHG methylation and 7214 overlapped regions that contained at least $10 \% \mathrm{CHH}$ methylation in wild-type individuals (Supplementary Data 3). To determine how many of these regions are susceptible to losing non-CG methylation if CG methylation is first depleted, we created a frequency distribution of $\mathrm{mCHG}$ and $\mathrm{mCHH}$ levels in wild-type and epimutagenized individuals (Fig. 1h, i). In total, 2341 and 3447 regions lost more than 10\% CHG methylation in 35S:TET11 and 35S:TET1-2, respectively, whereas 2475 and 3379 regions lost more than 5\% CHH methylation in 35S:TET1-1 and 35S: TET1-2, respectively. Regions that are susceptible to losses of CG and non-CG methylation in lines expressing hTET1cd share a substantial overlap with regions that lose non-CG methylation in met1 (Supplementary Fig. 1g, h). In total, 1708 (73.0\%) and 2386 $(69.2 \%)$ regions that have lost more than $10 \% \mathrm{mCHG}$ in $35 \mathrm{~S}$ :
TET1-1 and 35S:TET1-2 have reduced levels in met1, whereas $2013(81.3 \%)$ and $2563(75.9 \%)$ regions that have lost more than $5 \% \mathrm{mCHH}$ in 35S:TET1-1 and 35S:TET1-2 have reduced levels in met 1 . As crop genomes have a greater number of loci targeted for silencing by $\mathrm{CG}, \mathrm{CHG}$, and $\mathrm{CHH}$ methylation when compared to A. thaliana, ectopic expression of hTET1cd is likely a viable approach for the creation epiRILs ${ }^{18}$.

TET1-mediated variation of CHG methylation. Mutations in met1 also result in hypermethylation of CHG sites in gene bodies due to the loss of methylation in the seventh intron of the histone 3 lysine 9 (H3K9) demethylase, increase in bonsai methylation 1 $(I B M 1)^{19-22}$. This results in alternative splicing of IBM1, ultimately producing a non-functional gene product (IBM1-S), which results in ectopic accumulation of di-methylation of $\mathrm{H} 3 \mathrm{~K} 9$ 
(H3K9me2) throughout the genome ${ }^{23}$. As in met1, the seventh intron of IBM1 was hypomethylated in 35S:TET1-1, 35S:TET1-2, and an additional two lines, 35S:TET1-2 ${ }^{\mathrm{T} 5}$ and 35S:TET1-2 ${ }^{\mathrm{T} 6}$, which were propagated for an additional two and three generations, respectively (Fig. 2a). The increased abundance of IBM1-S transcript was confirmed by RT-qPCR in line 35S:TET1-2 $2^{\mathrm{T} 6}$ (Supplementary Fig. 2a), leading to CHG hypermethylation at gbM loci (Fig. 2b). Further quantitative analysis revealed extensive variation in genome-wide gains and losses of CHG methylation in these two lines, $\sim 1.8 \mathrm{Mb}$ and $2.3 \mathrm{Mb}$ of additional $\mathrm{CHG}$ methylation, respectively (Fig. $2 c$ and Supplementary Fig. $2 b, c$ ). To test the impact of a reduction in functional IBMI on H3K9me2, we performed chromatin immunoprecipitation (ChIP) against $\mathrm{H} 3 \mathrm{~K} 9 \mathrm{me} 2$ in $35 \mathrm{~S}$ :TET1-2 ${ }^{\mathrm{T} 6}$, which revealed a subtle increase in $\mathrm{H} 3 \mathrm{~K} 9 \mathrm{me} 2$ in gbM loci that possessed $\mathrm{CHG}$ hypermethylation (Fig. 2d).

To further characterize regions of differential CHG methylation, identified CHG DMRs in line 35S:TET1-2 ${ }^{\mathrm{T} 5}$ were categorized into discrete groups based on their DNA methylation status in wild-type individuals. Of the 9917 CHG DMRs identified, 1460 were in loci that are defined as gbM in wildtype individuals, 584 were in unmethylated regions, and 6940 of them were in RdDM-like regions (Fig. 2e-g). Interestingly, in line 35S:TET1-2 ${ }^{\mathrm{T} 5}, 1409$ (96.5\%) of the CHG DMRs in gbM-like loci gained CHG hypermethylation, whereas 2680 (38.6\%) of the CHG DMRs in RdDM-like regions lost CHG, in contrast to 825 (11.9\%) RdDM-like regions that gained CHG methylation. Lastly, there were $503(86.1 \%)$ loci that are unmethylated in wild-type individuals that gain $\mathrm{CHG}$ methylation as well as $\mathrm{CG}$ and $\mathrm{CHH}$ methylation in the epimutagenized lines (Fig. 2e-g). These results reveal that methods for epimutagenesis can result in both losses and gains in DNA methylation genome wide.

To characterize the effect of hTET1cd-induced methylome changes on gene expression, we performed RNA-sequencing (RNA-seq) on leaf tissue of wild-type, 35S:TET1-1 and 35S:TET12. Compared to wild-type plants, 629 and 736 upregulated genes were identified in 35S:TET1-1 and 35S:TET1-2, respectively, with 176 and 260 genes overlapping with identified CG DMRs. A total of 1277 and 1428 downregulated genes were identified and 268 and 324 of them overlapped with CG DMRs. There was a high level of overlap in transcriptome changes seen in 35S:TET1-1 and 35S:TET1-2 compared to met 1 and $i b m 1$ (Fig. $2 \mathrm{~h}, \mathrm{i}$ ). Of the genes upregulated in met 1, 36.8 and $38.7 \%$ overlapped with upregulated genes in 35S:TET1-1 and 35S:TET1-2, respectively (Supplementary Fig. 2d). An even greater overlap was observed with downregulated genes in met 1 , as 60.1 and $65.2 \%$ overlapped with downregulated genes in 35S:TET1-1 and 35S:TET1-2, respectively (Supplementary Fig. 2e). These results reveal that hTET1cd expression in $A$. thaliana is a viable approach for accessing hidden sources of allelic variation by inducing expression variation.

TET1 expression leads to a delay in the floral transition. In the transgenic plants that were used for WGBS, we observed a delay in the developmental transition from vegetative to reproductive growth (Fig. 3a, b). We hypothesized that the observed lateflowering phenotype was associated with the demethylation of the FLOWERING WAGENINGEN (FWA) locus, as is observed in met 1 mutants 24,25 . A closer inspection of the DNA methylation status of this locus revealed that DNA methylation was completely abolished, as was methylation at adjacent $\mathrm{CHG}$ and $\mathrm{CHH}$ sites (Fig. 3c). As in met1, the loss of methylation at the FWA locus was associated with an increase in FWA expression (Fig. 3d), which is known to cause a delay in flowering by restricting the movement of the florigen signal, $F T$, to the shoot a

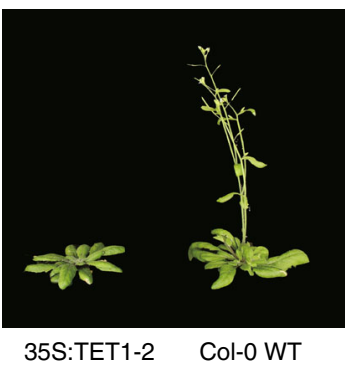

b

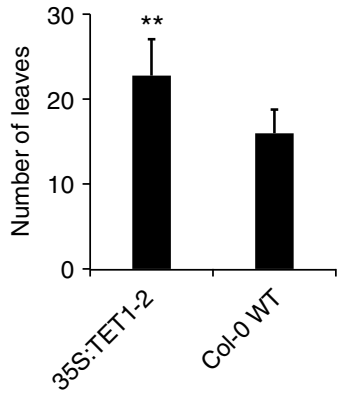

C

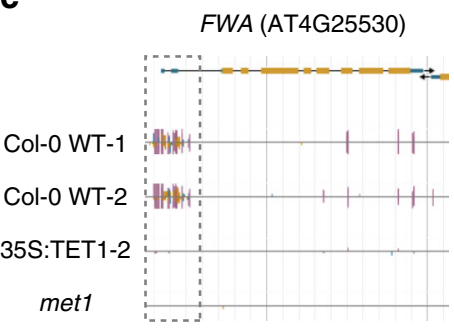

d

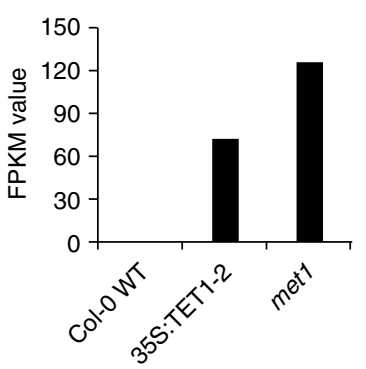

Fig. 3 35S:TET1 plants have a delayed flowering phenotype. a Photographs of one 35S:TET1-2 transgenic plant and Col-0 WT plant and $\mathbf{b}$ corresponding number of rosette leaves. Error bars indicate s.d. (**t-test, $p$ value $<0.01$ ). c Genome browser view of FWA (AT4G25530). Both CG and non-CG DNA methylation are depleted from the 5'UTR in 35S:TET1-2 plants (purple vertical lines $=C G$ methylation, blue vertical lines $=$ CHG methylation, and gold vertical lines $=\mathrm{CHH}$ methylation). $\mathbf{d}$ Expression level (FPKM) of FWA

$\operatorname{apex}^{26}$. These results demonstrate that expression of hTET1cd leads to phenotypic variation by abolishing methylation at some regions in all sequence contexts (CG, $\mathrm{CHG}$, and $\mathrm{CHH}$ sites).

TET1-mediated demethylation is transgenerationally inherited. To assess the stability and inheritance of TET1-mediated demethylation, T1 individuals expressing 35S:TET1 were self-fertilized, allowing for the loss of the hTET1cd transgene due to allelic segregation. Unexpectedly, transgene-free 35S:TET1-1 T2 individuals (35S:TET1-1.3-TET1, 35S:TET1-1.4-TET1, and 35S:TET1$1.5^{-T E T 1}$ ) exhibited a reversion to a normal flowering phenotype, and genome-wide methylation levels closely resembled that of wild-type individuals (Fig. 4a). WGBS on T2 individuals retaining the transgene revealed similar levels of CG methylation as the T1 parent (35S:TET1-1.1 $1^{+T E T 1}$ and 35S:TET1-1.2+TET1). The methylation level of CGs at genes and transposons revealed that demethylation of gbM loci was partially inherited in transgenefree T2 individuals, whereas active remethylation was found at transposons in the same individuals (Fig. $4 \mathrm{~b}-\mathrm{f}$ ). These results indicate that an active process likely in the meristem and/or germline is counteracting the activity of the hTET1cd transgene $^{27}$. To quantify how many regions were susceptible to the loss of non-CG methylation, a DMR analysis was conducted for each 35S:TET1-1 T2 individual. A total of 655 and 659 CHG hypomethylated regions were identified in $\mathrm{T} 2$ lines retaining the transgene. In contrast, 211, 155 and 199 hypomethylated regions were identified in three transgene-free $\mathrm{T} 2$ individuals, respectively (Fig. 4g).

To determine if increased expression of hTET1cd in meristematic tissue would increase the likelihood of germline transmittance of demethylation to transgene-free progeny, transgenic $A$. thaliana plants were generated expressing a previously described 
a

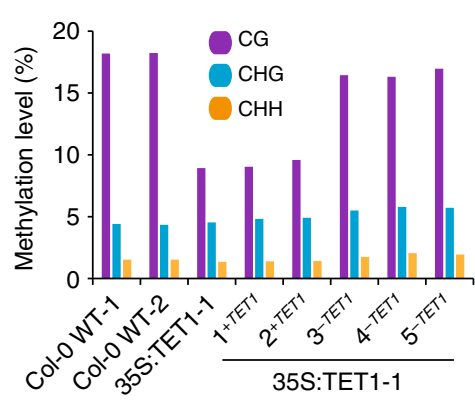

d

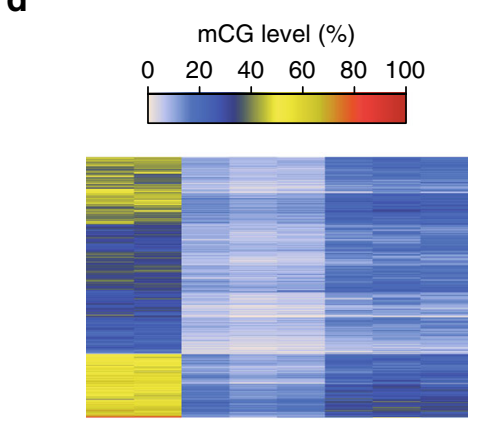

e

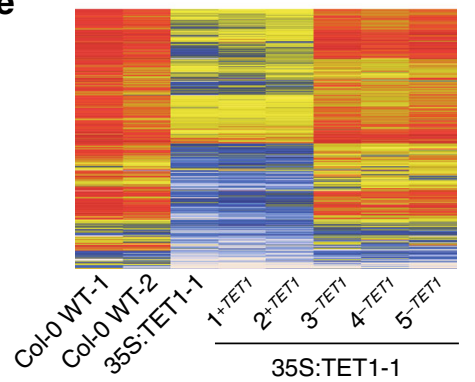

b

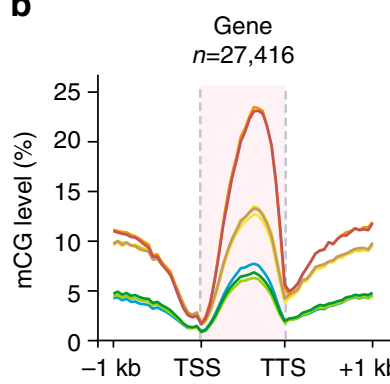

C

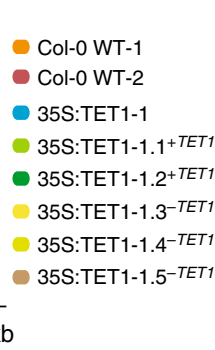

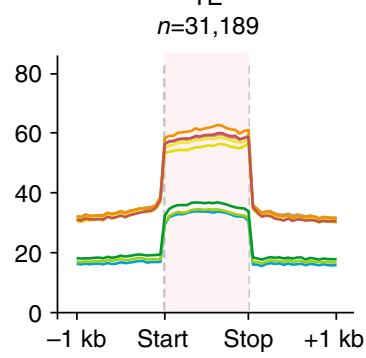

f

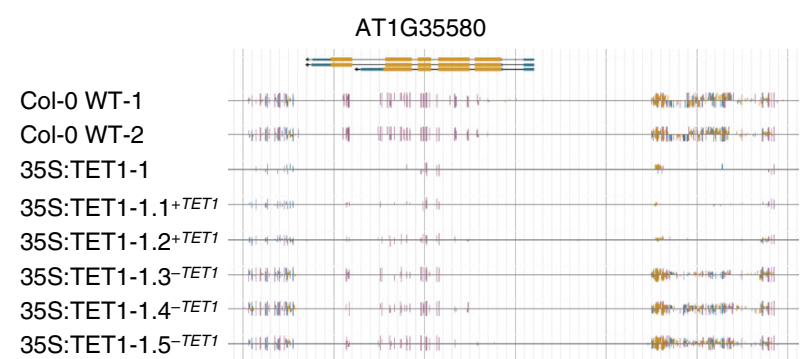

g

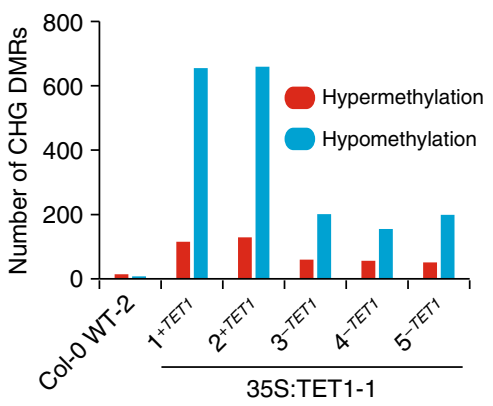

Fig. 4 Transgenerational demethylation profile of 35S:TET1 individuals. a Bar plots of global methylation levels in two Col-0 WT replicates and 35S:TET1-1 plants. Metagene plots of $\mathrm{mCG}$ level across $\mathbf{b}$ gene bodies and $\mathbf{c}$ transposable elements. Heat map of $\mathrm{mCG}$ level of $\mathbf{d}$ all gbM genes and $\mathbf{e}$ transposable elements with $>20 \% \mathrm{mCG}$ in wild type. $\mathbf{f}$ Genome browser view of a methylome profile of a representative region of the A. thaliana genome in $35 \mathrm{~S}$ :TET1-1 individuals. $\mathbf{g}$ Number of identified CHG DMRs in 35S:TET1 T2 plants. These DMRs were defined relative to Col-0 WT-1, as Col-0 WT-2 DMRs were used to assess background

superfolder GFP (sfGFP) hTET1cd fusion, under control of the $A$. thaliana ACTIN 2 (ACT2) promoter (ACT2:sfGFP-hTET1cd), which is known to have activity in all tissues of juvenile plants, including meristematic tissue ${ }^{28}$. Translation and nuclear localization of the sfGFP-TET1cd fusion protein was confirmed in young cotyledons using confocal microscopy (Supplementary Fig. 3a). T1 populations transformed with ACT2:sfGFP-hTET1cd exhibited a 27-fold increase in later flowering compared to $35 \mathrm{~S}$ : hTET1cd, indicating high activity of the sfGFP-TET1cd fusion protein in A. thaliana (Fig. 5a). To assess the variation between lines, we performed WGBS on four independent ACT2:TET1 T1 lines (Supplementary Fig. 4a-c). Differential levels of global CG demethylation were observed in these four lines, further confirming that plants subjected to epimutagenesis can possess different degrees of demethylation The expression of the sfGFPhTET1cd transgene was also confirmed by RT-qPCR in select lines (Supplementary Fig. 4d). Subsequent DMR analysis revealed 68,260 CG DMRs, 9235 CHG DMRs, and 2793 CHH DMRs between these lines (Supplementary Data 4-6), a drastic increase in DMRs compared to those within siblings in 35S:TET1 lines (Fig. 4g).
To further assess the inheritance of the demethylation pattern as a result of epimutagenesis, we selected T2 progeny of a late flowering ACT2:TET1-1.2-TET1 individual containing and lacking the transgene. WGBS data from these two individuals was used to confirm the presence/absence of the sfGFP-hTET1cd transgene (Supplementary Fig. 3d, e). Individuals retaining and lacking the transgene due to allelic segregation both exhibited a lateflowering phenotype (31 and 28 leaves upon flowering in ACT2:TET1-1.1 $1^{+ \text {TET1 }}$ and ACT2:TET1-1.2-TET1, respectively), ectopic expression, and loss of DNA methylation of FWA (Supplementary Fig. 3b, c). WGBS on these two individuals revealed a reduction in CG methylation that was maintained and stably inherited irrespective of transgene presence, confirming the high activity of demethylation in ACT2:TET1 lines compared to 35S:TET1 lines (Fig. 5b-f). It is unclear exactly why the lateflowering phenotype was stably inherited in the T2 individuals without the transgene in the ACT2-driven lines versus the 35Sdriven lines, although it is likely a combination of promoter strength and cell type specificity. For stable inheritance of demethylation and the late-flowering phenotype, TET1 activity would be required in the meristematic and/or germline cells. Collectively, these results demonstrate the stable inheritance of 

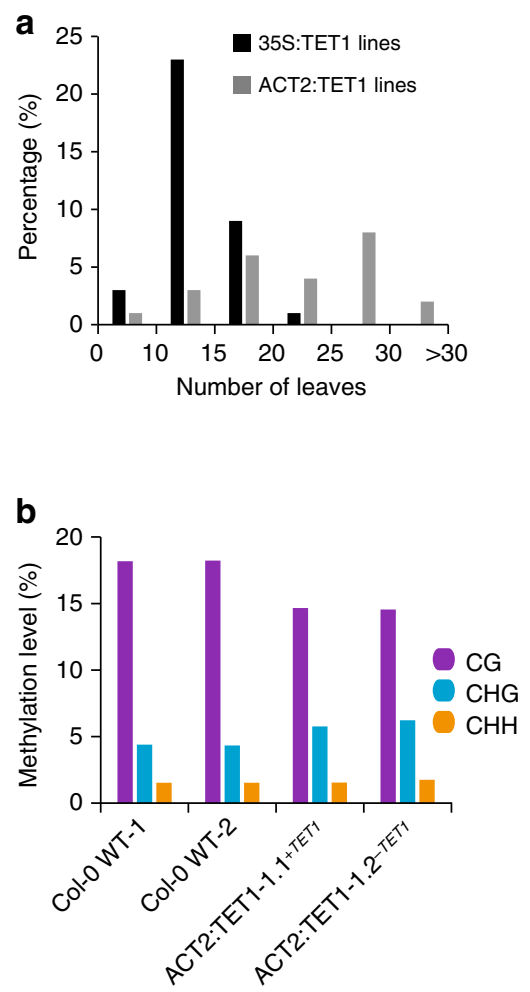

C

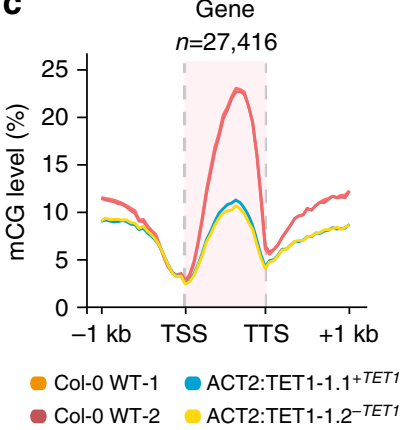

d

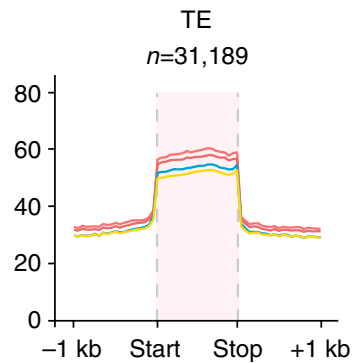

e

mCG level (\%)

020406080100

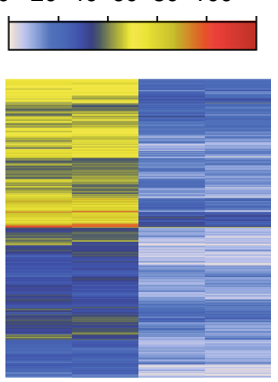

f

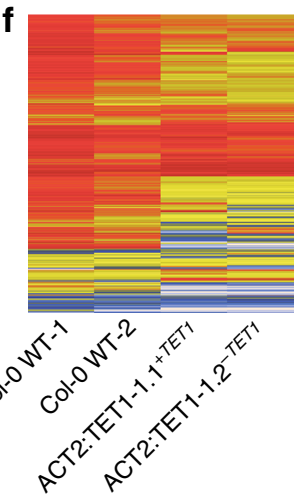

Fig. 5 Demethylation profile of ACT2:TET1 individuals. a Bar plot of number of rosette leaves in 35S:TET1 and ACT2:TET1 T1 individuals upon flowering. b Bar plot of global methylation levels in two Col-0 WT replicates and two ACT2:TET1-1 T2 plants. Metagene plots of mCG level across $\mathbf{c}$ gene bodies and $\mathbf{d}$ transposable elements. Heat map of $\mathrm{mCG}$ level of $\mathbf{e}$ all gbM genes and $\mathbf{f}$ transposable elements with $>20 \%$ mCG in wild type

TET1-mediated demethylation and a delayed floral transition in the absence of the transgene.

\section{Discussion}

The discovery that expression of the catalytic domain of the human TET1 protein in A. thaliana leads to widespread loss of CG methylation enables the creation epimutants without the need for methyltransferase mutants, which often causes lethality in crops. In addition to epimutagenesis, hTET1cd could be used in combination with sequence-specific DNA-binding proteins such as dCas9 to direct DNA demethylation in plant genomes, as has been demonstrated in mammalian systems ${ }^{29-33}$. The stable meiotic inheritance of DNA methylation states in flowering plant genomes provides a stark contrast to the inheritance of DNA methylation in mammalian genomes, where genome-wide erasure of DNA methylation and reprogramming occurs each generation $^{34}$. This property of flowering plant genomes makes them ideal targets of induced epialleles, as once a new methylation state occurs it is often inherited in subsequent generations. Application of epimutagenesis and the use of TET-mediated engineering of DNA methylation states in economically and agriculturally significant plant species will be an interesting area of future investigation.

\section{Methods}

Synthesis and cloning of the human TET1 catalytic domain. A human TET1 catalytic domain (hTET1cd) sequence (residues 1418-2136) was synthesized by GenScript, and moved to a plant transformation compatible vector (pMDC32) using LR clonase from Life Technologies as per the manufacturer's instructions (catalog \#11791100). ACT2:sfGFP-hTET1cd was subcloned by Genscript in the pMDC32 vector background using the sfGFP-TET1cd fragment from Addgene plasmid \#82561. The ACT2 promoter sequence was kindly provided by Dr. Richard Meagher.
Plant transformation and screening. The hTET1cd sequence in the pMDC32 vector was transformed into Agrobacterium tumefaciens strain $\mathrm{C} 58 \mathrm{C} 1$ and plated on LB-agar supplemented with kanamycin $(50 \mu \mathrm{g} / \mathrm{mL})$, gentamicin $(25 \mu \mathrm{g} / \mathrm{mL})$, and rifampicin $(50 \mu \mathrm{g} / \mathrm{mL})$. A single kanamycin-resistant colony was selected and used to start a 250-mL culture in LB Broth Miller liquid media supplemented with gentamicin $(25 \mu \mathrm{g} / \mathrm{mL})$, kanamycin $(50 \mu \mathrm{g} / \mathrm{mL})$, and rifampicin $(50 \mu \mathrm{g} / \mathrm{mL})$, which was incubated for 2 days at $30^{\circ} \mathrm{C}$. Bacterial cells were pelleted by centrifugation at $4000 \mathrm{rpm}$ for $30 \mathrm{~min}$ and the supernatant decanted. The remaining bacterial pellet was re-suspended in $200 \mathrm{~mL}$ of 5\% sucrose with $0.05 \%$ Silwet L77. Plant transformation was performed using the floral dip method described by Clough and Bent $^{35}$. Seeds were collected upon senescence and transgenic plants were identified via selection on $1 / 2$ LS plates supplemented with Hygromycin B $(25 \mu \mathrm{g} / \mathrm{mL})$. 35S: TET1-1 is a T1 individual, 35S:TET1-2 is a T3 plant, 35S:TET1-3 is a T4 plant. All transgenic individuals chosen for analysis contain independent insertions of hTET1cd and are not the result of single-seed decent unless otherwise noted.

DNA and RNA isolation. A. thaliana leaf tissue was flash-frozen and finely ground to a powder using a mortar and pestle. DNA extraction was carried out on all samples using the DNeasy Plant Mini Kit (Qiagen), and the DNA was sheered to $\sim 200$ bp by sonication. RNA was isolated from finely ground flash-frozen leaf tissue using Trizol (Thermo Scientific). For RT-qPCR, RNA was further treated with TURBO $^{\mathrm{m}}$ DNase (Thermo Scientific) according to the manufacturer's instructions. One microgram of RNA was subsequently reverse transcribed with M-MuLV reverse transcriptase according to the manufacturer's instructions (NEB). RT-qPCR was used to analyze cDNA populations using PP2AA-3 (AT1G13320) as an endogenous control, and was performed on a Roche LIghtCycler 480 instrument using SYBR Green detection chemistry. The genes assayed by this method were IBM1-S, IBM1-L, FWA and sfGFP-hTET1cd. Primers used for RT-qPCR were designed using PrimerQuest from Integrated DNA Technologies (www.idtdna.com/ PrimerQuest/). Primer sequences used for RT-qPCR: PP2AA-3-F: 5'-AATGAGGCAGAAGTTCGGATAG-3', PP2AA-3-R: 5'-CAGGGAAGAATGTGCTGGATAG3', ibm1s-F: 5'-TCTTTCTTCTAAGTCTGTCCATTCT-3', ibm1s-R: 5'-GTGACCGATTAGGAAATGGTATCT-3', ibm1L-F: 5'-CCGAAGCCAAAGTGGAGATA-3', ibm1L-R: 5'-CTTCCTCTTCCGTAGACTTCTTT-3', FWA-F: 5'-CAA-

GATGGTGGAAGGATGAGAA-3', FWA-R: 5' -CTCTGTTCTTCAGTGGGATGAG-3', sfGFP-hTET1cd-F: 5'-CAAAGATGACGGGACCTACAA-3', sfGFPhTET1cd-R: 5'-GTACTCGAGTTTGTGTCCAAGA-3'. 
Library construction. Genomic DNA libraries were prepared following the MethylC-seq protocol without use of the bisulfite conversion step. MethylC-seq libraries were prepared as previously described in ref. ${ }^{36}$. Briefly, genomic DNA was sonicated to $200 \mathrm{bp}$ using a Covaris S-series focused ultrasonicator, and endrepaired using End-It DNA end-repair kit (Epicentre). End-repaired DNA was subjected to A-tailing using Klenow $3^{\prime}-5^{\prime}$ exo ${ }^{-}$(NEB) and ligated to methylated adapters using T4 DNA ligase (NEB). Ligated DNA was subsequently bisulfite converted using the EZ DNA methylation-Gold kit as per the manufacturer's instructions and amplified using KAPA HiFi uracil + Readymix Polymerase. RNAseq libraries were constructed using Illumina TruSeq Stranded RNA LT Kit (Illumina, San Diego, CA) following the manufacturer's instructions with limited modifications. The starting quantity of total RNA was adjusted to $1.3 \mu \mathrm{g}$, and all volumes were reduced to a third of the described quantity. TAB-seq libraries were prepared as previously described in ref. ${ }^{16}$. Briefly, genomic DNA was glucosylated and oxidized using T4- $\beta$ GT (NEB) and recombinant mTET1. Standard bisulfite treatment is then performed using the MethylCode Bisulfite Conversion kit (Thermo Fisher Scientific).

ChIP library preparation. Leaves were treated with formaldehyde to covalently link protein to DNA, washed several times with distilled water, patted dry, and ground into fine powder in liquid nitrogen. Chromatin was extracted with a series of extraction buffers and sonicated. The final chromatin solution was incubated overnight with anti-H3K9me2 antibody (Cell Signaling Technology, 9753S)-coated dynabeads protein A (Life Technologies, 10002D) to precipitate the immune complex. After a few washes, the immune complex was eluted and incubated at $65^{\circ}$ in the presence of a high concentration of $\mathrm{NaCl}$ in a water-bath overnight. After degrading the proteins with proteinase K, DNA was recovered by phenol/chloroform/isoamyl alcohol extraction followed by ethanol precipitation. The DNA pellet was then dissolved in $30 \mu \mathrm{l}$ of nuclease-free water.

Sequencing. Illumina sequencing was performed at the University of Georgia Genomics Facility using an Illumina NextSeq 500 instrument. For MethylC-seq and TAB-seq, raw reads were trimmed for adapters and preprocessed to remove low-quality reads using cutadapt 1.9.dev1 ${ }^{37}$. For RNA-seq and ChIP-seq, these processes were carried out by Trimmomatic v0.32 ${ }^{38}$. The mutant allele of met 1 is met1-3 and methylome data was downloaded under accession GSE39901. The mutant allele of $i b m 1$ is $i b m 1-6$.

MethyIC-seq data processing. Qualified reads were aligned to the A. thaliana TAIR10 reference genome as described in ref. ${ }^{39}$. Chloroplast DNA (which is fully unmethylated) was used as a control to calculate the sodium bisulfite reaction nonconversion rate of unmodified cytosines. All conversion rates were $>99 \%$ (Supplementary Table 1). The list of gbM genes used in this study was previously curated $^{18}$. Heat maps were clustered by complete linkage method conducted by $\mathrm{R}$ (https://www.r-project.org). All methylation levels reported in all analyses are presented as differences in absolute values, including defining DMRs and calculating hyper/hypomethylated regions. The only exception is in the comparison of mCG loss between gbM, where we used a percentage difference.

RNA-seq data processing. Qualified reads were aligned to the A. thaliana TAIR10 reference genome using TopHat v2.0.13 ${ }^{40}$ (Supplementary Table 2). Gene expression values were computed using Cufflinks v2.2.1 $1^{41}$. Genes determined to have at least twofold $\log _{2}$ expression changes by Cufflinks and passed tests were identified as differentially expressed genes. The Col-0 wild-type transcriptomes were downloaded using data from accession GSE75071.

TAB-seq data processing. Qualified reads were aligned to the A. thaliana TAIR10 reference genome using Methylpy as described in ref. ${ }^{39}$. A modified lambda DNA sequence was used to assess the quality of prepared libraries. In the added lambda sequence, all non-CG cytosines are unmethylated and CG cytosines are methylated to $5 \mathrm{mC}$. The "non-conversion" rate is used to measure the rate of non-CG cytosines failing to be converted to thymines after bisulfite treatment. The " $5 \mathrm{mC}$ nonconversion" is used to estimate the $5 \mathrm{mCG}$ cytosines failing to be converted to thymines after TET treatment (Supplementary Table 3).

ChIP-seq data analysis. Qualified reads were aligned to the A. thaliana TAIR10 reference genome using Bowtie 1.1.1 with following parameters: bowtie - $\mathrm{m} 1$-v2 --best --strata --chunkmbs 1024 -S. Aligned reads were sorted using samtools v 1.2 and clonal duplicates were removed using SAMtools version 0.1.19 (Supplementary Table 4).

Metaplot analysis. For metaplot analyses, twenty $50 \mathrm{bp}$ bins were created for both upstream and downstream regions of gene bodies/TEs. Gene bodies/TE regions were evenly divided into 20 bins. Weighted methylation levels were computed for each $\operatorname{bin}^{42}$.
DMR analysis. Identification of DMRs was performed as described in ref. ${ }^{43}$ and adjusted $p$ value (Benjamini-Hochberg correction) 0.05 was adopted as the cutoff. Only DMRs with at least five DMSs (differential methylated sites) and a 10\% absolute methylation level difference within each DMR were reported and used for subsequent analysis. For coverage calculations, each sample was combined with two Col-0 WT replicates to identify DMRs. Each sample was compared with both Col-0 WT replicates separately and for a DMR to be identified it must have been identified in both comparisons. Absolute methylation differences of $\pm(50 \%$ for CG, $10 \%$ for $\mathrm{CHG}$ and $\mathrm{CHH}$ ) were defined as hyper/hypomethylation, respectively. DMRs overlapping regions with $\mathrm{mCG} \geq 5 \%, \mathrm{mCHG}$ and $\mathrm{mCHH} \geq 1 \%$ in both two Col-0 WT replicates were defined as RdDM-like regions. DMRs overlapping regions with $\mathrm{mCG} \geq 5 \%$, $\mathrm{mCHG}$ and $\mathrm{mCHH}<1 \%$ in both two Col- 0 WT replicates were defined as gbM regions. DMRs overlapping regions with all three contexts less methylated at less than $1 \%$ in both Col- 0 WT replicates were defined as unmethylated regions. Overlap comparisons were performed using bedtools v2.26.0 ${ }^{44}$.

Data availability. The data generated from this study have been uploaded to Gene Expression Omnibus (GEO) database and can be retrieved through accession number GSE93024.

Received: 15 May 2017 Accepted: 2 February 2018

Published online: 01 March 2018

\section{References}

1. Law, J. A. \& Jacobsen, S. E. Establishing, maintaining and modifying DNA methylation patterns in plants and animals. Nat. Rev. Genet. 11, 204-220 (2010).

2. Johannes, F. et al. Assessing the impact of transgenerational epigenetic variation on complex traits. PLoS Genet. 5, e1000530 (2009).

3. Reinders, J. et al. Compromised stability of DNA methylation and transposon immobilization in mosaic Arabidopsis epigenomes. Genes Dev. 23, 939-950 (2009).

4. Cortijo, S. et al. Mapping the epigenetic basis of complex traits. Science 343, 1145-1148 (2014).

5. Bewick, A. J. et al. On the origin and evolutionary consequences of gene body DNA methylation. Proc. Natl Acad. Sci. USA 113, 9111-9116 (2016).

6. Cortijo, S., Wardenaar, R., Colome-Tatche, M., Johannes, F. \& Colot, V. Genome-wide analysis of DNA methylation in Arabidopsis using MeDIPChip. Methods Mol. Biol. 1112, 125-149 (2014).

7. Reinders, J. et al. Genome-wide, high-resolution DNA methylation profiling using bisulfite-mediated cytosine conversion. Genome Res. 18, 469-476 (2008).

8. Hofmeister, B. T., Lee, K., Rohr, N. A., Hall, D. W. \& Schmitz, R. J. Stable inheritance of DNA methylation allows creation of epigenotype maps and the study of epiallele inheritance patterns in the absence of genetic variation. Genome Biol. 18, 155 (2017).

9. Li, Q. et al. Genetic perturbation of the maize methylome. Plant Cell $\mathbf{2 6}$ 4602-4616 (2014).

10. $\mathrm{Hu}, \mathrm{L}$. et al. Mutation of a major CG methylase in rice causes genome-wide hypomethylation, dysregulated genome expression, and seedling lethality. Proc. Natl Acad. Sci. USA 111, 10642-10647 (2014).

11. Tahiliani, M. et al. Conversion of 5-methylcytosine to 5hydroxymethylcytosine in mammalian DNA by MLL partner TET1. Science 324, 930-935 (2009).

12. Pastor, W. A. et al. Genome-wide mapping of 5-hydroxymethylcytosine in embryonic stem cells. Nature 473, 394-397 (2011).

13. Ito, $\mathrm{S}$. et al. Role of Tet proteins in $5 \mathrm{mC}$ to $5 \mathrm{hmC}$ conversion, ES-cell selfrenewal and inner cell mass specification. Nature 466, 1129-1133 (2010).

14. Hollwey, E., Watson, M. \& Meyer, P. Expression of the C-terminal domain of mammalian TET3 DNA dioxygenase in Arabidopsis thaliana induces heritable methylation changes at rDNA loci. Adv. Biosci. Biotechnol. 7, 243-250 (2016).

15. Erdmann, R. M., Souza, A. L., Clish, C. B. \& Gehring, M. 5hydroxymethylcytosine is not present in appreciable quantities in Arabidopsis DNA. G3 (Bethesda) 5, 1-8 (2014).

16. Yu, M. et al. Tet-assisted bisulfite sequencing of 5-hydroxymethylcytosine. Nat. Protoc. 7, 2159-2170 (2012).

17. Stroud, H., Greenberg, M. V., Feng, S., Bernatavichute, Y. V. \& Jacobsen, S. E. Comprehensive analysis of silencing mutants reveals complex regulation of the Arabidopsis methylome. Cell 152, 352-364 (2013).

18. Niederhuth, C. E. et al. Widespread natural variation of DNA methylation within angiosperms. Genome Biol. 17, 194 (2016).

19. Saze, H., Shiraishi, A., Miura, A. \& Kakutani, T. Control of genic DNA methylation by a jmjC domain-containing protein in Arabidopsis thaliana. Science 319, 462-465 (2008). 
20. Miura, A. et al. An Arabidopsis jmjC domain protein protects transcribed genes from DNA methylation at CHG sites. EMBO J. 28, 1078-1086 (2009).

21. Rigal, M., Kevei, Z., Pelissier, T. \& Mathieu, O. DNA methylation in an intron of the IBM1 histone demethylase gene stabilizes chromatin modification patterns. EMBO J. 31, 2981-2993 (2012).

22. Lister, R. et al. Highly integrated single-base resolution maps of the epigenome in Arabidopsis. Cell 133, 523-536 (2008).

23. Rigal, M., Kevei, Z., Pélissier, T. \& Mathieu, O. DNA methylation in an intron of the IBM1 histone demethylase gene stabilizes chromatin modification patterns. EMBO J. 31, 2981-2993 (2012).

24. Soppe, W. J. et al. The late flowering phenotype of fwa mutants is caused by gainof-function epigenetic alleles of a homeodomain gene. Mol. Cell 6, 791-802 (2000).

25. Finnegan, E. J., Peacock, W. J. \& Dennis, E. S. Reduced DNA methylation in Arabidopsis thaliana results in abnormal plant development. Proc. Natl Acad. Sci. USA 93, 8449-8454 (1996).

26. Ikeda, Y., Kobayashi, Y., Yamaguchi, A., Abe, M. \& Araki, T. Molecular basis of late-flowering phenotype caused by dominant epi-alleles of the FWA locus in Arabidopsis. Plant Cell Physiol. 48, 205-220 (2007).

27. Baubec, T., Finke, A., Scheid, O. M. \& Pecinka, A. Meristem-specific expression of epigenetic regulators safeguards transposon silencing in Arabidopsis. EMBO Rep. 15, 446-452 (2014).

28. An, Y. Q. et al. Strong, constitutive expression of the Arabidopsis ACT2/ACT8 actin subclass in vegetative tissues. Plant J. 10, 107-121 (1996).

29. Choudhury, S. R., Cui, Y., Lubecka, K., Stefanska, B. \& Irudayaraj, J. CRISPRdCas9 mediated TET1 targeting for selective DNA demethylation at BRCA1 promoter. Oncotarget 7, 46545-46556 (2016).

30. Maeder, M. L. et al. Targeted DNA demethylation and activation of endogenous genes using programmable TALE-TET1 fusion proteins. Nat. Biotechnol. 31, 1137-1142 (2013).

31. Vojta, A. et al. Repurposing the CRISPR-Cas9 system for targeted DNA methylation. Nucleic Acids Res. 44, 5615-5628 (2016).

32. Mendenhall, E. M. et al. Locus-specific editing of histone modifications at endogenous enhancers. Nat. Biotechnol. 31, 1133-1136 (2013).

33. Liu, X. S. et al. Editing DNA methylation in the mammalian genome. Cell 167, 233-247.e217 (2016)

34. Heard, E. \& Martienssen, R. A. Transgenerational epigenetic inheritance: myths and mechanisms. Cell 157, 95-109 (2014).

35. Clough, S. J. \& Bent, A. F. Floral dip: a simplified method for Agrobacteriummediated transformation of Arabidopsis thaliana. Plant J. 16, 735-743 (1998).

36. Urich, M. A., Nery, J. R., Lister, R., Schmitz, R. J. \& Ecker, J. R. MethylC-seq library preparation for base-resolution whole-genome bisulfite sequencing. Nat. Protoc. 10, 475-483 (2015).

37. Martin, M. Cutadapt removes adapter sequences from high-throughput sequencing reads. EMBnet J. 17, 10-12 (2011).

38. Bolger, A. M., Lohse, M. \& Usadel, B. Trimmomatic: a flexible trimmer for Illumina sequence data. Bioinformatics 30, 2114-2120 (2014)

39. Schmitz, R. J. et al. Epigenome-wide inheritance of cytosine methylation variants in a recombinant inbred population. Genome Res. 23, 1663-1674 (2013).

40. Kim, D. et al. TopHat2: accurate alignment of transcriptomes in the presence of insertions, deletions and gene fusions. Genome Biol. 14, R36 (2013).

41. Trapnell, C. et al. Transcript assembly and quantification by RNA-Seq reveals unannotated transcripts and isoform switching during cell differentiation. Nat. Biotechnol. 28, 511-515 (2010).

42. Schultz, M. D., Schmitz, R. J. \& Ecker, J. R. 'Leveling' the playing field for analyses of single-base resolution DNA methylomes. Trends Genet. 28, 583-585 (2012).
43. Schultz, M. D. et al. Human body epigenome maps reveal noncanonical DNA methylation variation. Nature 523, 212-216 (2015).

44. Quinlan, A. R. \& Hall, I. M. BEDTools: a flexible suite of utilities for comparing genomic features. Bioinformatics 26, 841-842 (2010).

\section{Acknowledgements}

We thank Nathan Springer for comments and discussions on this study as well as the Georgia Genomics \& Bioinformatics Core and the Georgia Advanced Computing Resource Center for technical support. This work was supported by the National Science Foundation (MCB-1650331), by The Pew Charitable Trusts and by the Office of the Vice President of Research at UGA to R.J.S. C.H. was supported by the National Institutes of Health (NIH HG006827) and is an investigator of the Howard Hughes Medical Institute. W.T.J. was supported by a Scholars of Excellence Fellowship from the University of Georgia and National Institute of General Medical Sciences of the National Institutes of Health award number T32GM007103. The content is solely the responsibility of the authors and does not necessarily represent the official view of the National Institutes of Health.

\section{Author contributions}

R.J.S. conceived the project. L.J., W.T.J. and R.J.S. designed the experiments. L.J., W.T.J., X.S., L.H. and C.H. performed the research. L.J., W.T.J. and R.J.S. analyzed the data. R.J.S wrote the paper. All authors discussed the results and commented on the manuscript.

\section{Additional information}

Supplementary Information accompanies this paper at https://doi.org/10.1038/s41467 018-03289-7.

Competing interests: R.J.S. has a provisional patent pending for the development of this technology as it applies to epigenome engineering of plants, and declares no other competing interests. The remaining authors declare no competing interests.

Reprints and permission information is available online at http://npg.nature.com/ reprintsandpermissions/

Publisher's note: Springer Nature remains neutral with regard to jurisdictional claims in published maps and institutional affiliations.

Open Access This article is licensed under a Creative Commons Attribution 4.0 International License, which permits use, sharing, adaptation, distribution and reproduction in any medium or format, as long as you give appropriate credit to the original author(s) and the source, provide a link to the Creative Commons license, and indicate if changes were made. The images or other third party material in this article are included in the article's Creative Commons license, unless indicated otherwise in a credit line to the material. If material is not included in the article's Creative Commons license and your intended use is not permitted by statutory regulation or exceeds the permitted use, you will need to obtain permission directly from the copyright holder. To view a copy of this license, visit http://creativecommons.org/ licenses/by/4.0\%.

(C) The Author(s) 2018 